\title{
Improving Representation of Skin of Color in a Medical School Preclerkship Dermatology Curriculum
}

\author{
Yusef Yousuf $^{1} \cdot$ Jaime C. Yu ${ }^{2}$ \\ Accepted: 17 November 2021 / Published online: 30 November 2021 \\ (c) The Author(s) under exclusive licence to International Association of Medical Science Educators 2021
}

\begin{abstract}
Anti-Black racism has contributed to significant disparities in health status for Black individuals in Canada. Dermatology is one area where these health disparities are evident. Lack of appropriate medical education regarding dermatologic conditions in persons of color has been associated with worse health outcomes. This project improved representation in a preclerkship dermatology medical school curriculum, through a constructivist approach, by adding images and discussion points of skin diseases in persons of color to existing teaching sessions. Student evaluations demonstrated strong agreement with improved exposure to images of dark skin and improved comfort in identifying skin conditions in persons of color. This intervention represents an effective approach to updating representation in the dermatology curriculum.
\end{abstract}

Keywords Undergraduate medical education $\cdot$ Dermatology $\cdot$ Diversity $\cdot$ Preclerkship

\section{Background}

The turbulent events of 2020, from the global COVID-19 pandemic to worldwide protests for the Black Lives Matter movement, have highlighted the longstanding institutional racism within the Canadian healthcare system. Specifically, anti-Black racism has been noted as contributing to significant disparities in socioeconomic status and health status for the Black community in Canada [1]. Dermatology is one particular area of North American medicine where these health disparities are evident. Black patients routinely report dissatisfaction with their dermatologists' lack of knowledge regarding their unique skin presentations, and specialized knowledge and focus on care of Black skin and hair has been noted to improve care satisfaction [2,3]. Lack of education and knowledge regarding dermatologic conditions in skin of color has also been associated with worse health outcomes. A study in the USA found that Black and Hispanic patients had significantly more advanced stage of melanoma at

Jaime C. Yu

jaime.yu@ualberta.ca

1 Faculty of Medicine and Dentistry, MD Program, University of Alberta, Edmonton, Canada

2 Faculty of Medicine and Dentistry, Department of Medicine, Division of Physical Medicine and Rehabilitation, and MD Program, University of Alberta, Edmonton, AB, Canada presentation, and thus poorer outcomes, compared to White patients, with the greatest disparity between non-Hispanic Black persons and non-Hispanic White individuals [4]. The degree of representation and diversity within medical education curriculum and resources has been identified as an issue, with the lack of darker skin in medical textbooks and journals ultimately harming patient outcomes [5-7]. Dermatology education and research in Canada must expand to include greater representation of Black, Indigenous, and People of Color (BIPOC) patient populations to address these health inequities. This paper describes our constructivist learning approach to improve student knowledge of skin diseases in persons of color. Constructivism is based on the premise that students learn best when exposed to new information that builds on previous learning [9]. We built on students existing knowledge of common skin conditions in fair-skinned persons by introducing learning objectives centered around these same conditions in darker skinned persons.

\section{Activity}

To improve the representation within our local undergraduate dermatology medical school curriculum, strategic changes were implemented within the preclerkship dermatology course. Dermatology is delivered as a 1-week block 
of content within the Musculoskeletal (MSK) System course in the second year (preclerkship) of a 4-year Medical Doctor degree program at our institution. This preclerkship curriculum included didactic lectures, one small-group case-based discussion session with practicing dermatologists, three problem-based learning sessions with clinical or non-clinical facilitators, and one team-based learning session led by the MSK course faculty coordinator and the dermatology week faculty coordinator [8].

Similar to other medical school curricula in North America, there historically has not been any specific focus and a limited scope of exposure to dermatological conditions in darker skinned individuals within this course. The vast majority of images used in the preclerkship dermatology curriculum was of Caucasian patients. To improve student competency, strategic updates were made to the existing curriculum during the 2020-2021 academic year to improve representation of darker skin tones. Using the existing curriculum, opportunities to add clinical imagery and discussion points for all covered dermatological conditions were identified by an expert dermatologist, a student from the local Black Medical Student Association, and the course coordinator. Images and discussion prompts were added to student readings and handouts for case-based discussions and problem-based learning sessions, and lecturers for other topics were asked to voluntarily add selected images and discussion points relevant to their topic. Specific learning objectives were added to small-group sessions to increase opportunities to discuss the differences in presentations, responses to treatment, and potential complications of skin conditions in dark-skinned individuals. The detailed changes are outlined in Table 1. In addition, all small-group facilitator orientation sessions included instructions to the faculty to review the new learning objectives and to ensure there was space and time for discussion on differing presentations of skin conditions in different skin tones, with additional information provided in facilitator handouts.

Measures of effectiveness regarding the updates to the dermatology curriculum were included in the standard course evaluation conducted at the end of the week, with additional questions added for this academic year, as detailed in Table 2. Individual sessions were not evaluated on a standalone basis. Students rated these statements on a score of 1 to 5 , with 1 indicating strongly disagree and

Table 1 Strategic updates and innovations to preclerkship dermatology curriculum

\section{Type of learning session}

Lectures

\section{Case-based discussion session}

Session traditionally covered eczema, acne, warts, skin cancers

Problem-based learning sessions

Sessions traditionally worked through presentation of undifferentiated papulosquamous rash, with thorough discussion of psoriasis by end of week

Team-based learning (TBL) session
Updates to curriculum

- Lecturers asked to include representative images of skin conditions in both light and dark skin where possible

- More specific focus on different presentations of skin conditions highlighted in introduction lecture at beginning of week and in summary lecture at end of week

- Added images of dark-skinned individuals with atopic dermatitis, acne, warts, and skin cancers to traditional light-skinned clinical images

- Added learning objectives to discuss different characteristics of skin conditions in dark skin (e.g., grey versus red colored rash, pigmentation changes)

- Added specific content on presentation of skin cancers (basal cell carcinoma, malignant melanoma) in Black individuals, and how presentation differs from light-skinned individuals

- Clinical presentation and progression of the problem case was unchanged, and remained a White female with new onset psoriasis

- Added specific addition of learning objectives to discuss presentation of psoriasis and how it differs in persons of color, impact of topical steroids in light vs dark skin

- Added side-by-side images of plaque psoriasis and Koebner phenomenon in light and dark skin, to demonstrate erythematous vs violaceous appearance

- Updated all images to provide contrasting examples of papulosquamous rashes across numerous differential diagnoses, including lichen planus, pityriasis rosea, and secondary syphilis

- Added facilitated discussion points on differences in rash appearance, potential pigmentation changes due to rash

- Added specific question to weekly quiz (low-stakes assessment) on impact of topical corticosteroid treatment in light-skinned vs darkskinned individuals

- Changed previous question on malignant melanoma to have specific focus on this condition in Black individuals 
Table 2 Dermatology week student evaluation results

\begin{tabular}{|c|c|}
\hline Evaluation question & $\begin{array}{l}\text { Mean student } \\
\text { response } \\
\text { scores } \\
N=168 \\
M(S D)\end{array}$ \\
\hline The dermatology week provided exposure to images of skin conditions in a variety of skin types & $4.6(0.6)$ \\
\hline I feel more comfortable identifying skin conditions in persons of color after this week & $4.3(0.8)$ \\
\hline $\begin{array}{l}\text { The case-based learning sessions (CBL), discovery learning (DL), and team-based learning (TBL) were effective in providing } \\
\text { increased exposure to skin conditions in a variety of skin types }\end{array}$ & $4.4(0.7)$ \\
\hline The textbook resources on dermatology in skin of color were helpful for my studying & $4.3(0.8)$ \\
\hline I feel that the dermatology curriculum provides me with core competencies and is adequate for my future needs & $4.2(0.9)$ \\
\hline The dermatology week met my expectations & $4.3(0.8)$ \\
\hline
\end{tabular}

5 indicating strongly agree. Mean agreement scores were calculated for each statement, with higher scores indicating better agreement.

\section{Results}

Evaluation response rate was $100 \%$ (168/168). All evaluation questions showed strong agreement or positive responses from the student perspective. Specifically, students rated 4.6/5.0 agreement that the updated curriculum provided exposure to images of skin conditions in a variety of skin types, and 4.3/5.0 agreement that they felt more comfortable identifying skin conditions in persons of color after the week of content. At the end of the dermatology week, student performance on the individual TBL quiz showed that $68 \%$ of students correctly answered a question on topical corticosteroid side effects in dark skin, and $90 \%$ correctly answered a question on malignant melanoma in a Black patient. This improved to $89 \%$ for the topical corticosteroid question and $100 \%$ for the malignant melanoma question on the subsequent group TBL quiz, where students were able to discuss and answer together.

\section{Discussion}

Institutional racism within medical education must be stated transparently and acknowledged, in order to move forward with necessary curriculum and programmatic changes for the improvement of health disparities amongst the BIPOC patient populations in Canada. The changes made to our dermatology curriculum were received positively by students, with the majority of students indicating improved exposure to and improved comfort with identifying skin conditions in persons of color. The changes implemented were targeted and strategic and worked within the exiting dermatology curriculum. Large-scale overhaul or curriculum renewal was not required to achieve improvements in student exposure to skin conditions in different skin tones.

Unfortunately, the objective evaluation of student performance was limited. Only two out of 17 questions on the weekly team-based learning quiz were focused on specific dermatologic issues in Black patients, with the remaining questions more generalized to dermatology content through the week as well as a few questions on non-dermatology material (e.g., anatomy and embryology). Nonetheless, most students were able to answer the dermatology questions correctly, with further improvements after the group discussions. In addition to the curriculum changes, we provided students with resources on dermatology in skin of color which they reported to be helpful (Table 1). We did not measure if these resources were accessed by students or how frequently. No narrative comments on specific sessions from students were available.

Our intervention represents a simple, yet effective approach to improving representation within the dermatology curriculum. This was accomplished without considerable disruption to the existing curriculum and provides a basis for further improvements and ongoing curricular updates. Through a constructivist approach, we built on students' previous knowledge of dermatology and added new information on skin diseases in persons of color to the curriculum. Students were able to compare skin diseases between fair and dark-skinned persons in various learning modalities. Learning is more meaningful when new knowledge is linked to students' previous knowledge [9]. Much of medicine is based on pattern recognition. However, this pattern recognition may fail when treating persons of color. We hope that by improving the "mental models of disease presentation" in our students, we can produce stronger clinicians that are better able to treat persons of color.

The lack of representation in dermatology medical education is a major issue as numerous diseases have cutaneous manifestations that differ in darker skin tones and thus can impact presentation and outcomes [3, 7]. Students' must be able to spot these differences to improve patient satisfaction and care. Prior works by Louie and Wilkes [6] and Adelekun 
et al. [7] have shown significant overrepresentation of light skin tone and underrepresentation of dark skin tone in medical textbook imagery. Curated and targeted integration of images and content thus must be done by curriculum developers and educators to provide improved representation for students. While these changes represent a step in the right direction, curriculum changes in dermatology need to be implemented at all Canadian medical institutions to improve patient satisfaction and reduce the significant health disparities.

Acknowledgements The authors would like to acknowledge Dr. John Elliott, Professor, Department of Medicine, Division of Dermatology, for his contributions as the faculty content expert in developing the curriculum updates implemented in this project.

Funding This project was funded internally by the University of Alberta, Faculty of Medicine and Dentistry, MD Program, Summer Studentship Program.

\section{Declarations}

Ethics Approval This project was a local education quality improvement project, reviewed by the University of Alberta Research Ethics Board coordinator, and ethics review was waived.

Consent to Participate Participants in this project completed all components as part of the usual educational process, with no additional elements requiring specific informed consent.

Consent for Publication Not applicable.

Conflict of Interest The authors declare no competing interests.

\section{References}

1. Dryden O, Nnorom O. Time to dismantle systemic anti-black racism in medicine in Canada. CMAJ. 2021;193(2):E55-7. https:// doi.org/10.1503/cmaj.201579.

2. Gorbatenko-Roth K, Prose N, Kundu RV, Patterson S. Assessment of black patients' perception of their dermatology care. JAMA Dermatol. 2019;155(10):1129-34. https://doi.org/10.1001/jamadermatol.2019. 2063.

3. Taylor SC. Meeting the unique dermatologic needs of black patients. JAMA Dermatol. 2019. https://doi.org/10.1001/jamadermatol.2019. 1963.

4. Hu S, Soza-Vento RM, Parker DF, Kirsner RS. Comparison of stage at diagnosis of melanoma among Hispanic, black, and white patients in Miami-Dade County, Florida. Arch Dermatol. 2006;142(6):704-8. https://doi.org/10.1001/archderm.142.6.704.

5. Perlman KL, Klein EJ, Park JH. Racial disparities in dermatology training: the impact on black patients. Cutis. 2020;106(6):300301. https://doi.org/10.12788/cutis.0135.

6. Louie P, Wilkes R. Representations of race and skin tone in medical textbook imagery. Soc Sci Med. 2018;202:38-42. https://doi. org/10.1016/j.socscimed.2018.02.023.

7. Adelekun A, Onyekaba G, Lipoff JB. Skin color in dermatology textbooks: an updated evaluation and analysis. J Am Acad Dermatol. 2021;84(1):194-6. https://doi.org/10.1016/j.jaad.2020. 04.084 .

8. van Diggele C, Burgess A, Mellis C. Planning, preparing and structuring a small group teaching session. BMC Med Educ. 2020;20(2):462. https://doi.org/10.1186/s12909-020-02281-4.

9. Dennick R. Constructivism: Reflections on twenty five years teaching the constructivist approach in medical education. Int $\mathbf{J}$ Med Educ. 2016;7:200-5. https://doi.org/10.5116/ijme.5763.de11.

Publisher's Note Springer Nature remains neutral with regard to jurisdictional claims in published maps and institutional affiliations. 